\title{
Ecological safety of buildings depending on the inaccuracies of engineering investigations
}

\author{
$S$. Chernyshov ${ }^{1}$, and $A$. Martynov ${ }^{1, *}$ \\ ${ }^{1}$ Moscow State University of Civil Engineering, Yaroslavskoe shosse, 26, Moscow, 129337, Russia
}

\begin{abstract}
The ecological safety of buildings and structures built on deformable soils depends largely on the settlement calculation. A classic example of unaccounted influence of differential settlement is the Leaning Tower of Pisa. Its analogues are numerous among the monuments of Russian architecture. The issue of modern builders to exclude the repetition of defects of old masters, who did not have calculating methods of soil mechanics. The accuracy of modern calculations largely depends on the accuracy of engineering investigations' materials. In the overall assessment of the system's work, the building-base is currently making a large inaccuracy in the incompleteness of survey information, especially definition the location of geological boundaries directly under the foundation. Two examples show the dependency of the geological soil profile inaccuracy on the distance between the wells and it gives a quantitative assessment of the relationship between the magnitude of the inaccuracy and the distance between the drilling wells of geologists. The numerical modeling method shows the influence of such an investigation inaccuracy on the result of the settlement calculation.
\end{abstract}

\section{Introduction}

Operating conditions and safety of buildings depends on the quality of the design of the building-environment system. Design and construction of such a system is possible only based on a detailed and comprehensive research of its natural component, in most of the builtup areas already modified previously completed construction. The quality of the environment defines the construction industry engineering investigations. In the article, using the example of prediction the natural and technical process of settlement, which defines its stability, we show that the quality of engineering investigations strongly affects the quality of the process prediction by methods of geomechanics.

One of the main parts of the environment is the geological medium, soils. The building is connecting with the surrounding geological medium by means of a foundation structure. Layers of soils, foundations on them and bearing structures of the building constitute a unified natural and technical system. During construction, part of the geological medium, receives the role of a bearing structure that works together with the rest of the building's loadbearing structures, and becomes the base. To ensure the normal operation of the basefoundation pair, engineering geological and geotechnical investigations are performing. In

\footnotetext{
*Corresponding author: martynov30am@gmail.com
} 
research, as in any process, performers inevitably admit inaccuracies. In engineering geological investigations, inaccuracies can be very significant due to the heterogeneity of the geological medium, namely, the variability of soil characteristics within a conventionally homogeneous layer, the tortuosity of the boundaries of the layers, the fluctuation of the ground water level. In the report, we show the magnitude of the inaccuracies in engineering geological investigations and assess their influence on the accuracy of the settlement calculation of the building. The magnitude of the settlement is the main numerical criterion for the quality of the building's connection with the geological medium. Technical standards of Russia [3] provide maximum permissible settlements, which are specifying separately for buildings of different design and number of storeys.

\section{Materials and methods}

In the report, two objects of research are considered. First, this is a real geological soil profile in the Nizhny Novgorod region of Russia, which for the first time revealed the magnitude of the inaccuracies in engineering geological investigation that influence the accuracy of settlement calculation of the building. Second, it is a calculated model of the base, on which also estimates of the inaccuracy in calculating the settlement are deriving for the first time, caused by the established investigation inaccuracies.

The geological soil profile (fig. 1) was studying in the building pit. The photo clearly shows the boundaries between a layer of clay, a homogeneous brown color and a layer of yellow sand under the clay. Deformation modulus of clay $10 \mathrm{MPa}$, modulus of deformation of sand $45 \mathrm{MPa}$. The boundary between such different compressible soils should be accurately showing in the calculated model. Above the clay lies a layer of technogenic bulk soils, for which the deformation modulus according to Russian standards is not indicating. Bulk soil cannot be leaving at the base of the responsible structure. Therefore, the accurate knowledge of the boundary is actual. The research of geological soil profile is aiming at determining the accuracy of the geological boundary at the geological soil profile.

To assess the influence of investigation inaccuracies, the researches were carrying out on a model of a layered base with a single foundation. The base consists of three layers of soil with different deformation characteristics. The layers are separating, as usual in the geological medium, by clear boundaries. As usual, they occur horizontally under the foundation. The compressibility of soils decreases from the upper layer to the lower one. The ratio of the deformation modulus of the upper layer to the underlying layer in a series of successively studied models is taking to be the corresponding series $1 / 2,1 / 3,1 / 4$.

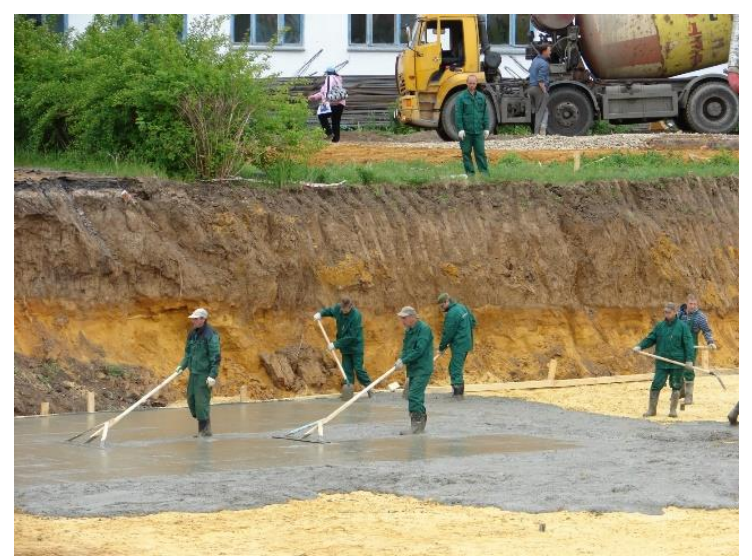

Fig. 1. Fragment of the soil profile in the building pit in the Nizhny Novgorod region of Russia. 


\section{Results}

Methodology and results of the research on the first object.

To define the magnitude of the inaccuracy of the boundary between the soils occurring on the engineering geological soil profiles, a panoramic photograph of the pit was using. Conditionally, two wells No. 1 and 2 were drilling at the edges of the panorama. The depths of occurrence at a given location of the boundary between clay and sand were defining from the well lines. On two points, the geological boundary is drawing in the form of a straight line, as geologists do in the construction of geological soil profiles. Well No. 3 was then drilling in the middle between the two extreme. It defines the true position of the geological boundary. The difference between the true value and the No. 1 and 2 wells found was 0.91 meters by the interpolation method. Further, increasing the number of wells and reducing the distance between them, we obtain a graph (fig.2) of the dependence of the geological boundary inaccuracy on the distance between the wells on this soil massif. A graph for the boundary between bulk clay and natural clay was also obtaining (fig. 3).

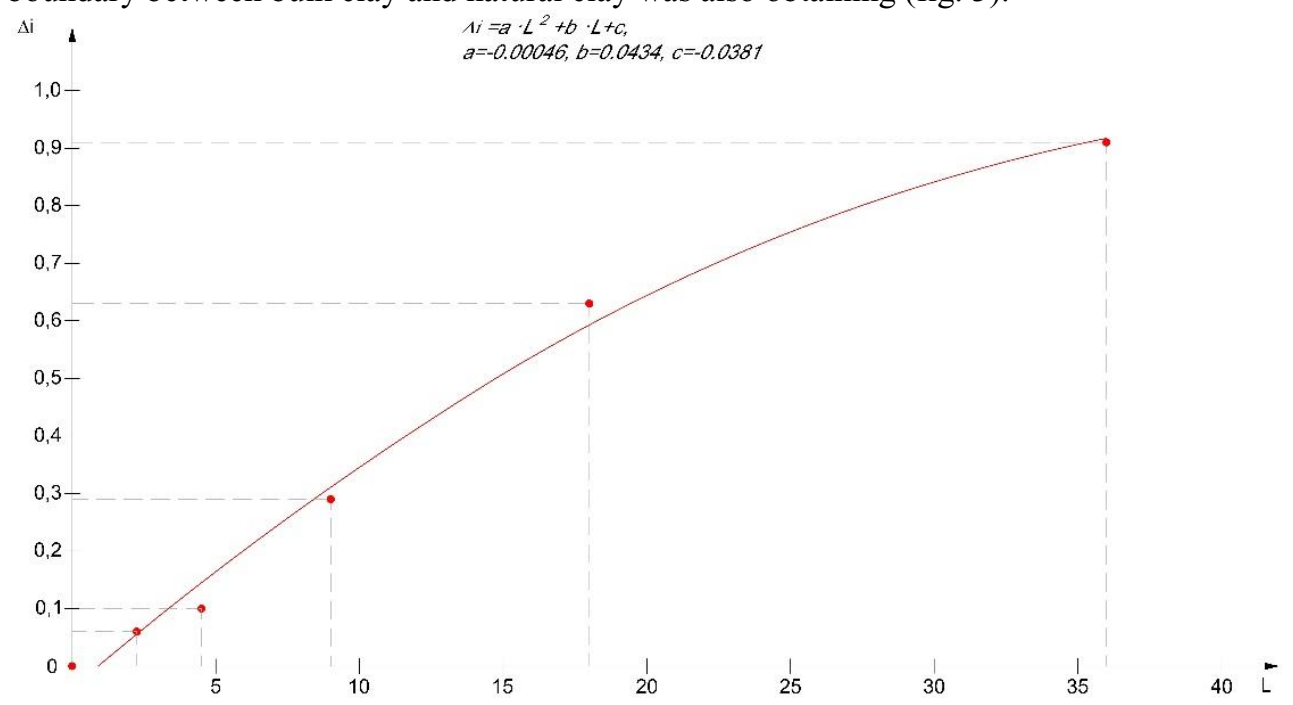

Fig. 2. Graph of dependence of the interpolation inaccuracy $(\Delta \mathrm{i})$ on the distance between the wells (L) for the boundary between the cover clays and neogene sands.

As can be seen from the graphs, the inaccuracy of the geological boundary at the geological soil profiles between the wells exceeds 0.5 meter with a distance between the wells of the order of 20 meters and exceeds 1 meter at a distance of 40-50 meters. Such distances between drilling wells are common in investigations, and therefore the found inaccuracies are common in investigations. When we first researched these inaccuracies, we give them a terminological name "inaccuracy of interpolation". Note that the found inaccuracy values are not universal, since the magnitude of the inaccuracy probably varies with the origin of the boundary. 


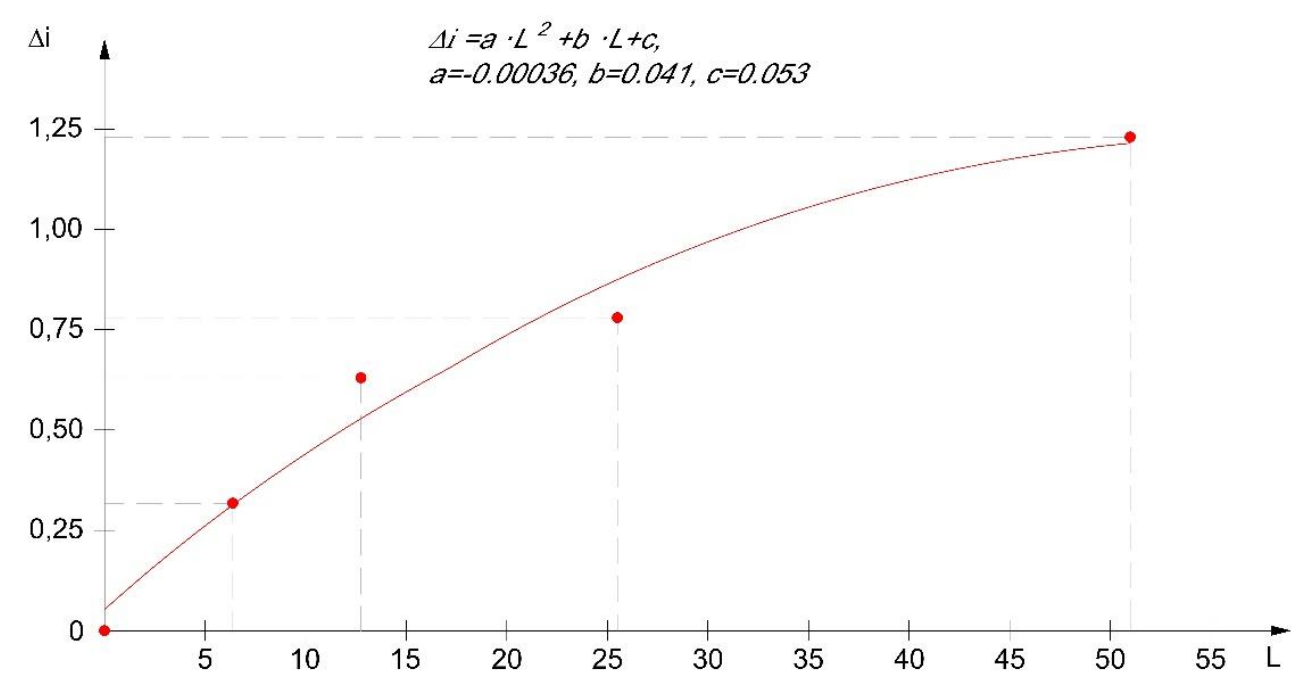

Fig. 3. Graph of the interpolation inaccuracy $(\Delta \mathrm{i})$ for the geological boundary of the "sole of technogenetics deposits" as a function of the distance between the wells (L) in the profile.

The methodology and results of the research on the second object.

Now we establish the influence of the interpolation inaccuracy on the result of the settlement calculation. To do this, we will perform a numerical experiment with the settlement calculation according to the true values of the position of the geological boundaries and from the values changed by the magnitude of the interpolation inaccuracy. The calculation is carrying out by the method of layerwise addition [3, 4].

The calculation model (fig. 4) consists of a single basement on a three-layer base. The third layer is at a depth of 5.2 meters from the footing level and is not shown in the figure, but it is involved in the calculation. We introduce the inaccuracy of geologists into the model, for which we change the position of the boundary of the 1 st and 2 nd layers by $0,15,0,25$, 0,50 and 1,00 meters. For the ratio of the deformation modulus of the layers E2 / E1 = 2, we obtain (fig. 4) a graph of the relative inaccuracy in the settlement calculation $(\Delta s / s)$ versus the boundary inaccuracy $\Delta \mathrm{h}$. In the second series of calculations, we take E2 / E1 $=3$. In the third series of calculations, we take E2 $/ \mathrm{E} 1=4$. As the difference between E1 and E2 increases, the inaccuracy in calculation significantly increases. At E2 / E1 = 4 and the real inaccuracy of the geological boundary $\Delta \mathrm{h}=1,00$ meter, the inaccuracy of calculation exceeds $50 \%$ of the exact predicted value of the settlement (fig. 5).

Analogous calculations with the displacement of the boundary between the second and third layers of the model showed an order of magnitude less inaccuracy in calculation due to the interpolation inaccuracy (table 1, table 2 and table 3 ). In the first case, the boundary was at a relative depth approximately equal to the width of the basement. In the second case, it lies about 4 times deeper. This greatly reduced the influence of the geological inaccuracy on the result of the settlement calculation. 


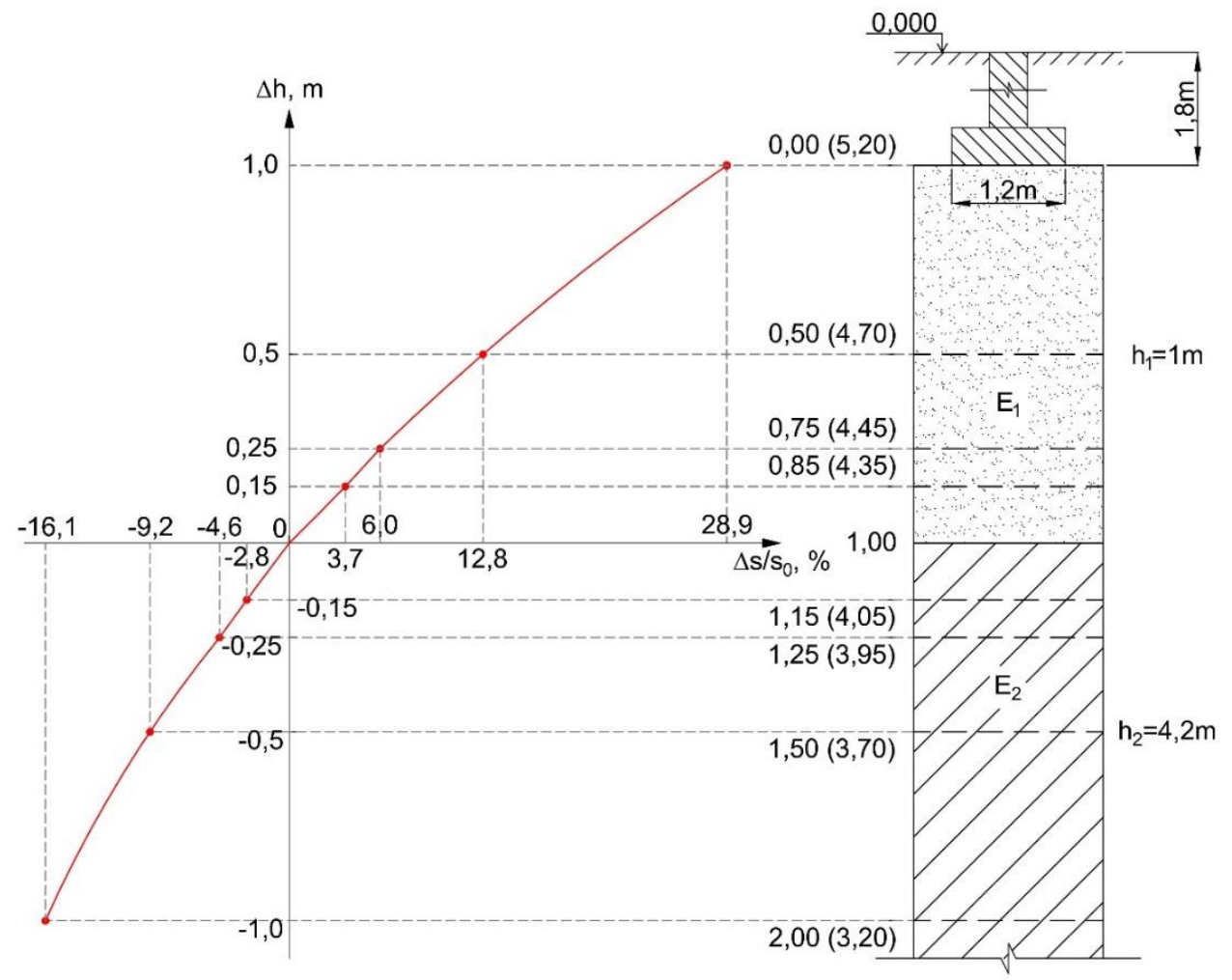

Fig. 4. Graph of the dependence of the relative inaccuracy in the settlement calculation $(\Delta \mathrm{s} / \mathrm{s}, \%)$ on the inaccuracy $(\Delta \mathrm{h}, \mathrm{m})$ on the upper geological boundary on the engineering geological soil profile when considering the deformation modulus of the layers E2 / E1 $=2,0$.

Table 1. The inaccuracy in settlement calculating of the building with the ratio of the deformation modulus of the layers of soil under the foundation base E3 / E2 $=2,0$ for various inaccuracies in the geological boundary located at a depth of 5.2 meters from the basement.

\begin{tabular}{|c|c|c|c|c|c|c|}
\hline \multirow{2}{*}{$\begin{array}{l}\text { Calcul } \\
\text { ation } \\
\text { № }\end{array}$} & \multirow{2}{*}{$\begin{array}{l}\text { Boundary } \\
\text { inaccuracy }\end{array}$} & \multicolumn{2}{|l|}{ Layer thickness, $\mathrm{m}$} & \multirow{2}{*}{$\begin{array}{l}\text { Calculated } \\
\text { settlement } \mathrm{s},\end{array}$} & \multicolumn{2}{l|}{$\begin{array}{l}\text { Settlement calculation } \\
\text { inaccuracy }\end{array}$} \\
\cline { 7 - 8 } & $\mathrm{h}, \mathrm{m}$ & $\mathrm{h}_{1}, \mathrm{~m}$ & $\mathrm{~h} 2, \mathrm{~m}$ & $\mathrm{~mm}$ & $\Delta \mathrm{s}, \mathrm{mm}$ & $\Delta \mathrm{s} / \mathrm{s}_{\mathrm{o}}, \%$ \\
\hline 1 & $-1,00$ & 3,20 & 7,00 & 21,10 & 0,70 & 3,3 \\
\hline 2 & $-0,50$ & 3,70 & 6,50 & 21,50 & 0,30 & 1,4 \\
\hline 3 & $-0,25$ & 3,95 & 6,15 & 21,70 & 0,10 & 0,5 \\
\hline 4 & $-0,15$ & 4,05 & 6,15 & 21,78 & 0,02 & 0,1 \\
\hline 5 & 0,00 & 4,20 & 6,00 & 21,80 & 0,00 & 0,0 \\
\hline 6 & 0,15 & 4,35 & 5,85 & 21,90 & $-0,10$ & $-0,5$ \\
\hline 7 & 0,25 & 4,45 & 5,75 & 22,00 & $-0,20$ & $-0,9$ \\
\hline 8 & 0,50 & 4,70 & 5,50 & 22,00 & $-0,20$ & $-0,9$ \\
\hline 9 & 1,00 & 5,20 & 5,00 & 22,30 & $-0,50$ & $-2,3$ \\
\hline
\end{tabular}




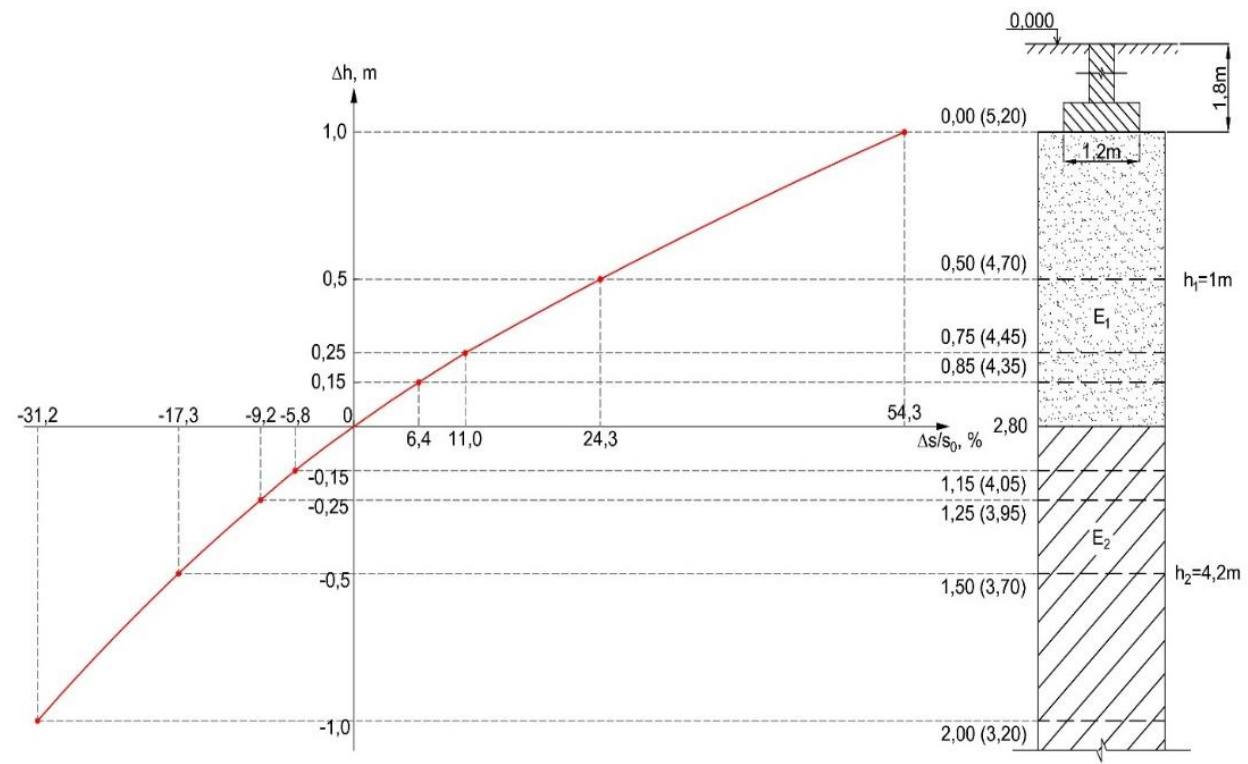

Fig. 5. Graph of the dependence of the relative inaccuracy in the settlement calculation $(\Delta \mathrm{s} / \mathrm{s}, \%)$ on the inaccuracy $(\Delta \mathrm{h}, \mathrm{m})$ of deposition of the upper geological boundary on the engineering geological soil profile with the ratio of the deformation modulus of the layers E2 / E1 $=4,0$.

Table 2. The inaccuracy in settlement calculating of the building with the ratio of the deformation modulus of the layers of soil under the foundation base E3 / E2 = 3,0 for various inaccuracies in the geological boundary located at a depth of 5.2 meters from the basement.

\begin{tabular}{|c|c|c|c|c|c|c|}
\hline \multirow{2}{*}{$\begin{array}{c}\text { Calculation } \\
\text { No }\end{array}$} & \multirow{2}{*}{$\begin{array}{c}\text { Boundary } \\
\text { inaccuracy } \\
\Delta \mathrm{h}, \mathrm{m}\end{array}$} & \multicolumn{2}{|c|}{$\begin{array}{c}\text { Layer } \\
\text { thickness, } \mathrm{m}\end{array}$} & \multirow{2}{*}{$\begin{array}{c}\text { Calculated } \\
\text { settlement s, mm }\end{array}$} & \multicolumn{2}{|c|}{$\begin{array}{c}\text { Settlement calculation } \\
\text { inaccuracy }\end{array}$} \\
\hline & & $\mathrm{h}_{2}, \mathrm{~m}$ & $\mathrm{~h}_{3}, \mathrm{~m}$ & & $\Delta \mathrm{s}, \mathrm{mm}$ & $\Delta \mathrm{s} / \mathrm{s}_{\mathrm{o}}, \%$ \\
\hline 1 & $-1,00$ & 3,20 & 7,00 & 20,90 & 0,80 & 3,7 \\
\hline 2 & $-0,50$ & 3,70 & 6,50 & 21,30 & 0,40 & 1,8 \\
\hline 3 & $-0,25$ & 3,95 & 6,15 & 21,60 & 0,10 & 0,5 \\
\hline 4 & $-0,15$ & 4,05 & 6,15 & 21,68 & 0,02 & 0,1 \\
\hline 5 & 0,00 & 4,20 & 6,00 & 21,70 & 0,00 & 0,0 \\
\hline 6 & 0,15 & 4,35 & 5,85 & 21,80 & $-0,10$ & $-0,5$ \\
\hline 7 & 0,25 & 4,45 & 5,75 & 21,90 & $-0,20$ & $-0,9$ \\
\hline 8 & 0,50 & 4,70 & 5,50 & 22,00 & $-0,30$ & $-1,4$ \\
\hline 9 & 1,00 & 5,20 & 5,00 & 22,30 & $-0,60$ & $-2,8$ \\
\hline
\end{tabular}


Table 3. The inaccuracy in settlement calculating of the building with the ratio of the deformation modulus of the layers of soil under the foundation base E3 / E2 $=4,0$ for various inaccuracies in the geological boundary located at a depth of 5.2 meters from the basement.

\begin{tabular}{|c|c|c|c|c|c|c|}
\hline \multirow{2}{*}{$\begin{array}{c}\text { Calculation } \\
\text { № }\end{array}$} & \multirow{2}{*}{$\begin{array}{c}\text { Boundary } \\
\text { inaccuracy } \\
\end{array}$} & \multicolumn{2}{|c|}{$\begin{array}{c}\text { Layer } \\
\text { thickness, } \mathrm{m}\end{array}$} & \multirow{2}{*}{$\begin{array}{c}\text { Calculated } \\
\text { settlement s, }\end{array}$} & \multicolumn{2}{|c|}{$\begin{array}{c}\text { Settlement } \\
\text { calculation } \\
\text { inaccuracy }\end{array}$} \\
& $\Delta \mathrm{h}, \mathrm{m}$ & $\mathrm{h}_{2}, \mathrm{~m}$ & $\mathrm{~h}, \mathrm{~m}$ & $\mathrm{~m}$ & $\Delta \mathrm{s}, \mathrm{mm}$ & $\Delta \mathrm{s} / \mathrm{s}_{\mathrm{o}}, \%$ \\
\hline 1 & $-1,00$ & 3,20 & 7,00 & 20,70 & 0,90 & 4,2 \\
\hline 2 & $-0,50$ & 3,70 & 6,50 & 21,20 & 0,40 & 1,9 \\
\hline 3 & $-0,25$ & 3,95 & 6,15 & 21,50 & 0,10 & 0,5 \\
\hline 4 & $-0,15$ & 4,05 & 6,15 & 21,58 & 0,02 & 0,1 \\
\hline 5 & 0,00 & 4,20 & 6,00 & 21,60 & 0,00 & 0,0 \\
\hline 6 & 0,15 & 4,35 & 5,85 & 21,80 & $-0,20$ & $-0,9$ \\
\hline 7 & 0,25 & 4,45 & 5,75 & 21,90 & $-0,30$ & $-1,4$ \\
\hline 8 & 0,50 & 4,70 & 5,50 & 22,00 & $-0,40$ & $-1,9$ \\
\hline 9 & 1,00 & 5,20 & 5,00 & 22,30 & $-0,70$ & $-3,2$ \\
\hline
\end{tabular}

\section{Discussion}

Experiments have shown that the inaccuracies in the geological boundaries at geological soil profiles, with a normal distance between the wells for exploration, can exceed \pm 1 meter from the true position of the boundary. To this inaccuracy, according to the rule for adding independent random variables, the inaccuracy in defining the boundary allowed at the drilling point should be adding [5]. In the design calculations, these inaccuracies of prospectors are not taking into account. Requirements for the accuracy of the boundaries are not formulating. "Designers perceive boundaries between layers, as something unmistakably established" [6]. Normative regulations for settlement calculating are not included in the margin because of this inaccuracy, since prior to this publication it was not known about the inaccuracy of interpolation. It is establishing here that the interpolation inaccuracy allowed in investigations can lead to an inaccuracy in calculating the construction-base system that exceeds half the calculated value.

Soil characteristics, the deformation modulus and soil density included in the settlement calculation are by their nature random variables. However, they are also taking into account without a safety factor for the soil according to [1]. The document is international, as it is officially accepting in Azerbaijan, Armenia, Kyrgyzstan, Moldova, Uzbekistan and Ukraine. The introduction of a margin on the soil into a characteristic of a general deformation modulus can lead to a refinement of the calculation by $5-15 \%$, depending on the number of tests and the natural heterogeneity of a particular layer of the calculation model. This random inaccuracy is independent of the inaccuracy caused by an inaccurate boundary. Inaccuracies can be summing according to the rule of adding random inaccuracies. The influence of the inaccuracy in defining the general deformation modulus will be insignificant after such addition. Geomechanics for many decades has been working on the creation of reliable methods for defining the general deformation modulus and it is defining quite accurately. The same is true with the definition of soil density - a characteristic that has a relatively small natural dispersion and is defining in the laboratory with an inaccuracy of 1-2\% [2] and therefore does not influence the accuracy of the settlement calculation. 


\section{Conclusions}

The ecological safety of buildings and structures erected on deformable soils depends to a large extent on the settlement calculation. A classic example of the influence of irregular settlement is the Pisa tower. Its analogues are numerous among the monuments of Russian architecture. The task of modern builders to exclude the repetition of mistakes of old masters not armed with the methods of soil mechanics. The accuracy of modern calculations largely depends on the accuracy of engineering investigations materials.

The analysis shows that when planning an engineering investigations, the volume of which is defined by the chief engineer of the project, it is necessary to increase the number of boring holes and sounding points for defining the position of the boundaries located directly under the foundation, as well as the boundaries with a contrast transition from the strongly compressible soil to weakly compressible. The latter type of boundaries can be successfully defining by an inexpensive and rapid method of sounding, if the weakly compressible soil is not a rocky soil.

\section{References}

1. Russian Standard GOST 20522-2012

2. V.V. Dmitriev, L.A. Yarg, Methods and quality of laboratory study of soils (KDU, Moscow, 2008)

3. Russian Standard SP 22.13330.2016

4. N.A. Tsytovich, Soil mechanics (short course). The fourth edition revised and enlarged (Higher School, Moscow, 1983)

5. S.N. Chernyshev, A.A. Krivoguzov, Engineering Survey 5, 22-27 (2017)

6. E.R. Chernyak, Engineering investigations, 1 (2009) 